\title{
INCLUSIÓN FINANCIERA Y EMPODERAMIENTO DE LA MUJER: UNA REVISIÓN CRÍTICA EN BASE A LA LITERATURA
}

\section{Ignacio E. Carballo*}

Pontificia Universidad Católica Argentina

$\triangle$ ignaciocarballo@uca.edu.ar

Recibido: 22 de octubre de 2019 Aceptado: 5 de enero de 2020

DOI: $10.46553 /$ colec.31.1.2020.p141-168

Resumen: El presente trabajo estudia la inclusión financiera y su rol como instrumento para reducir la inequidad de género. Se propone principalmente analizar la evidencia de impacto que sustenta los causales teóricos en torno a la inclusión financiera de la mujer como herramienta para la reducción de brechas mediante el empoderamiento de la misma. Para ello, se expone el fenómeno reciente de fomento a la inclusión financiera con enfoque de género y se plantean los causales teóricos que la justifican. Tras analizar la evidencia empírica que la sustenta, se denota un quiebre en los trabajos que han abordado la evaluación de programas de inclusión financiera con diseños metodológicos aleatorios y controlados (RCT) con respecto a evaluaciones previas a estos, cuyas conclusiones de impacto eran mayormente positivas. Se señala el valor de los primeros como únicos diseños metodológicos que pueden afirmar algún impacto causal y se destaca cómo la evidencia en estos estudios es nula o (en el mejor de los casos) mixta, poniendo en duda el impacto real de la inclusión financiera en el empoderamiento de la mujer.

Palabras clave: inclusión financiera: microfinanzas; género

\footnotetext{
* Ignacio E. Carballo es docente en la Pontificia Universidad Católica Argentina y miembro del Centro de Estudios de la Estructura Económica (CENES), Instituto de Investigaciones Económicas, CONICET, FCE-UBA.
} 


\begin{abstract}
This paper studies financial inclusion and its role as an instrument to reduce gender inequality. It is mainly proposed to analyze the evidence of impact that sustains the theoretical causes around the financial inclusion of women as a tool for the reduction of gaps through the empowerment of women. To this end, the recent phenomenon of promoting financial inclusion with a gender perspective is presented, as well as the theoretical causes that justify it. After analyzing the empirical evidence that supports it, a break in the work that has approached the evaluation of financial inclusion programs with random and controlled methodological designs (RCT) with respect to previous evaluations to these, whose conclusions of impact were mostly positive, is highlighted. It emphasizes the value of the former as the only methodological designs that can affirm any causal impact and highlights how the evidence in these studies is null or (at best) mixed, calling into question the real impact of financial inclusion on women's empowerment.
\end{abstract}

Keywords: Financial Inclusion; Microfinances; Gender

\title{
I. Introducción
}

Según ONU Mujeres, las inequidades de género se manifiestan en cada una de las dimensiones de los Objetivos de Desarrollo Sostenible (ODS). Ello es sumamente crítico, ya que las mujeres representan la mitad de la población del mundo $\mathrm{y}$, por ende, tienen la mitad del potencial humano (ONU Mujeres 2018).

De acuerdo al organismo, el empoderamiento de la mujer no solo es bueno por sí mismo desde una visión ética, al mejorar su bienestar personal, sino también que trae aparejados efectos positivos para la sociedad en su conjunto. En los términos de la Agenda 2030: una mujer que logra obtener ingresos regulares no sólo contribuye a reducir la pobreza (ODS Nro. 1), sino que también mejora, por ejemplo, la educación, salud y nutrición de quienes de ella dependen (ODS Nro. 2, 3 y 4) (Durrant y Sathar 2000; Lancaster et al. 2003; Joshi 2004; ONU Mujeres 2018).

No obstante, las mujeres enfrentan barreras legales, prejuicios y normas sociales más duras y más dificultades personales que los hombres en un 
amplio número de aspectos. Para dimensionar, a nivel mundial hay un $22 \%$ más de mujeres pobres que hombres en edades entre 25 y 34 años (ONU Mujeres 2018).

Entre otras brechas, las mujeres demuestran un menor acceso a recursos financieros, pero mejores tasas de cumplimiento. De acuerdo al Banco Mundial:

La inclusión financiera se refiere al acceso que tienen las personas y las empresas a una variedad de productos y servicios financieros útiles y asequibles que satisfacen sus necesidades -como pagos y transferencias, ahorro, seguros y crédito- y que son prestados de una manera responsable y sostenible. (Banco Mundial 2018)

En este trabajo se entenderá a la inclusión financiera como la creación, promoción y regulación de un ambiente financiero accesible, asequible y seguro para el conjunto de la sociedad. Tiene como fin promover el bienestar económico y la inclusión social a través de la oferta de servicios y productos financieros diseñados para satisfacer las necesidades de distintos sectores de la población (Carballo 2018, 2)

Cada vez más, la inclusión financiera se destaca como una prioridad en materia de desarrollo, para las autoridades, los organismos reguladores y las instituciones tanto a nivel mundial como nacional. Como factor clave para el desarrollo económico y la reducción de la pobreza, la inclusión financiera pasó a jugar un papel importante en la agenda mundial y de políticas públicas.

Para dar un ejemplo el Grupo Banco Mundial ha impulsado la iniciativa de lograr el "Acceso universal a los servicios financieros para el año 2020" (UFA 2020), y cuya meta principal es proporcionar acceso financiero a los 1.500 adultos excluidos del sistema financiero formal, entre los cuales más de la mitad son mujeres ${ }^{37}$. En la declaración presentada por el Grupo Banco Mundial se destaca los 25 países en desarrollo en donde viven más del 70\% de las personas no bancarizadas, poniendo foco a los avances que deberían

\footnotetext{
${ }^{37}$ Véase UFA2020 Overview: Universal Financial Access by 2020, disponible en: https://www.worldbank.org/en/topic/financialinclusion/brief/achieving-universalfinancial-access-by-2020
} 
lograrse para alcanzar las metas de inclusión financiera universal y colocando a los Gobiernos Nacionales como promotores y guías de políticas que acompañen las metas de inclusión. Asimismo, el G-20 se ha comprometido con la promoción de la inclusión financiera, apostando a los beneficios de las tecno finanzas digitales principalmente a través del uso de dispositivos móviles (GPFI 2016).

Inmersos en este fenómeno, la inclusión financiera se ha situado en el centro del empoderamiento económico de las mujeres y esgrimido como estandarte en el camino hacia la igualdad de género. Es por ello que, en búsqueda del fortalecimiento de sus capacidades y el desarrollo de la comunidad, desde los organismos multilaterales y cada vez más los distintos gobiernos, se denota de gran importancia garantizar y facilitar el acceso de la mujer a los servicios financieros. Se han elaborado servicios que especialmente apuntan a mujeres, no sólo por los mayores beneficios comparativos que reportan, sino también por un objetivo social: el de mejorar su bienestar y el de los miembros del hogar que ellas componen.

Según esta lógica, la participación activa de las mujeres en la inclusión económica y financiera generaría un efecto derrame de beneficios tangibles que van desde su propio hogar al resto de la comunidad. Por supuesto, el papel protagónico que ejerce la mujer en el bienestar de su familia, que la lleva muchas veces a ser la encargada de gestionar los recursos financieros fuera y dentro de la vivienda familiar, es un factor clave. Pero este factor es ampliamente discutido, justamente, por ser reflejo y consecuencia de la misma inequidad de género entorno al trabajo de cuidado derivado en las mujeres.

En esta línea, las brechas sociales, salariales y de género obstaculizan el logro de estos objetivos y constituyen barreras que multiplican las dificultades de crecimiento y desarrollo de las mujeres. El contexto desfavorable que debe enfrentar el género femenino se agrava ante condiciones de empleo injustas, depresiones económicas, estereotipos negativos de género, dispersión geográfica, ausencia de conciliación familiar y personal, falta de promoción en el trabajo y tareas no remuneradas en el hogar, entre otras. Es necesario superar una gran serie de desafíos en el logro de mujeres emprendedoras y/o empresarias.

A su vez, el concepto de empoderamiento está claramente vinculado con la noción de poder, pero la ausencia del mismo, el desempoderamiento y la 
vulnerabilidad de género en sus múltiples expresiones, reprimen y limitan los campos de acción y participación de las mujeres. Esta inequidad se extiende en todos los niveles de la sociedad, el empoderamiento de la mujer y su inclusión en el mundo debe ser abordado en pos de un bienestar general de la comunidad y no solo como cuestión de género.

En camino a mitigar la desigualdad de género y otorgar mayores herramientas financieras a las mujeres se deben abordar los desafíos que se presentan con cambios estructurales, económicos y culturales. Por ello este trabajo se centrará en el impacto que causan los programas que apuntan a una mayor inclusión financiera de las mismas, a través de ciertos servicios microfinancieros.

Para ello se entenderá que el empoderamiento es un proceso en el cual las mujeres mejoran sus posibilidades para realizar elecciones fundamentales para sus vidas en aspectos donde antes no podían hacerlo. Debe prestarse especial relevancia a que el empoderamiento de la mujer tiene una connotación especial respecto a otros grupos y es su carácter trasversal y con limitantes propias no presentes en estos otros, provocados esencialmente por la brecha en la dedicación al trabajo no remunerado o labores reproductivas.

Otra disyuntiva a plantearse en la revisión de este tema es cómo medir el impacto de una mayor inclusión en el empoderamiento de la mujer. A lo largo de la historia de la investigación sobre microfinanzas e inclusión financiera se han utilizado diversos métodos, cuya diferencia principal yace en la aleatoriedad en la asignación del servicio (o tratamiento). En el presente trabajo se hará un breve repaso sobre parte de la literatura previa al auge de este método, para luego describir algunos trabajos que hayan aplicado como método al Estudio Aleatoreo Controlado (Randomized Control Trial).

Los experimentos aleatorizados controlados han proliferado en este milenio, pero han llegado a conclusiones menos prometedoras sobre el impacto de las microfinanzas en general, sobre todo en el empoderamiento de la mujer. Aun así, varios son los trabajos donde ha habido efectos positivos (aunque más moderados) de las microfinanzas. Ello lleva a realizar la ya afirmada conclusión sobre que la inclusión financiera es una dimensión más dentro de los instrumentos utilizados para la reducción de la pobreza y mejora del bienestar de las personas, y que no debe tenérsela a ésta como 
"píldora mágica" contra esta problemática. La pobreza es multivariada en sus causas y en sus posibles soluciones.

Para abordar los objetivos descriptos, en la siguiente sección realizaremos un diagnóstico y definiremos nuestros conceptos de estudio. Seguidamente, en la tercera sección, realizaremos nuestro análisis de la literatura que ha buscado demostrar el impacto de la inclusión financiera en el empoderamiento de la mujer. Finalmente realizaremos esbozaremos nuestras conclusiones a modo de reflexiones finales.

\section{Diagnóstico y marco teórico}

\section{II.1 Dimensiones en la exclusión financiera de la mujer}

De acuerdo a los estudios realizados a nivel global, se estima que aproximadamente una de cada tres mujeres en el mundo está excluida del sistema financiero formal, es decir el 40\% de la población femenina (1.100 millones de mujeres), según Global Findex del Banco Mundial. En el 2017, la brecha de acceso a cuentas bancarias persiste en torno al 9\% para economías de bajos y medianos ingresos. Allí solo el 44\% de las mujeres asalariadas reportan recibir su sueldo en cuenta bancaria. A nivel global, los hombres representarían el $65 \%$ de los clientes, el $80 \%$ del volumen de préstamos y el $75 \%$ de los depósitos en entidades bancarias (Demirguc-Kunt et al. 2018)

Se estima entonces que las mujeres tienen un $7 \%$ menos de probabilidades de tener una cuenta bancaria que los hombres en los países desarrollados. Esta brecha financiera por razón de género aumenta entre los países más pobres, observándose que las mujeres de los hogares más pobres, aquellas que viven con menos de US\$2 al día, tienen un $18 \%$ menos de probabilidades que los hombres de ser titulares de una cuenta bancaria y un $17 \%$ menos probabilidades de haber recibido un préstamo de una institución financiera formal.

La exclusión financiera de las mujeres responde a numerosos desafíos que obstaculizan el poder lograr acceso universal a servicios financieros. A continuación, siguiendo el artículo de Yasmin Bin-Humam (2017), se 
analizará las principales restricciones a las que se enfrentan las metas de inclusión financiera de la mujer.

En primer lugar, identificar a las mujeres excluidas del sistema bancario presenta grandes dificultades. Entre ellas encontramos a los mecanismos de recolección de datos, como las encuestas a los hogares que son costosas, principalmente por tratarse de poblaciones muy dispersas y requieren mucho tiempo; limitando de esta manera el acceso a la información y representando una barrera para el revelamiento formal de los datos. Esto impide la distinción de los segmentos de la población que están excluidos del sector financiero, y en consecuencia el diseño y la implementación de los esfuerzos necesarios para a ampliar el acceso financiero. De hecho, el reciente trabajo realizado por The Economist Intelligence Unit (2019) encontró que, aunque tres cuartas partes de 55 países emergentes recopilan algunos datos sobre inclusión financiera, solo una cuarta parte desglosa esos datos por género. Un dato a resaltar es que Chile se destaca como el único país que ha recopilado estos datos segregados por género durante más de una década (EIU 2019)

La obtención de información es también muy relevante para las instituciones financieras, quienes a la hora de brindar un servicio financiero deben evaluar la información crediticia de sus clientes. Estas desventajas de las mujeres en términos de recopilación de datos generan restricciones crediticias y evidencian una realidad: las mujeres obtienen menos créditos que los hombres.

De 173 economías analizadas por el Banco Mundial, 30 no cuentan con un registro público de información crediticia o una central de riesgo privada que abarque a más del $5 \%$ de la población. No registrándose, entre otros, los créditos otorgados por instituciones microfinancieras, cuyos prestatarios son en una gran proporción mujeres, dejando excluidos sus historiales de reembolso y repago (Banco Mundial 2016).

En segundo lugar, otro de los desafíos que encontramos, son las normas sociales las cuales constituyen una barrera al limitar la demanda de servicios financieros para las mujeres. Esto se debe a la costumbre de no esperar que las mujeres sean independientes en términos financieros y al poco o nulo incentivo de lograr ese objetivo. Los datos de la desigualdad por razón de género presentados en el informe "Mujer, Empresa y el Derecho" destacan entre otros la potestad legal del esposo en 18 economías, para impedir que 
la mujer trabaje, como también las restricciones legales que impiden a las mujeres abrir una empresa. Un contexto social desfavorable que reduce las probabilidades de que las mujeres alcancen las oportunidades que el sistema financiero ofrece (Banco Mundial 2016).

La importancia de la participación de la mujer en el hogar está vinculada en gran parte con la probabilidad de que tengan acceso a una cuenta bancaria. Según los datos de Global Findex, en las economías donde el esposo es quien tiene el derecho de administrar los bienes conyugales se observa una disminución de la proporción de mujeres con acceso a cuentas bancarias formales, ahorros o crédito; esta situación mejora en los países en donde existe un régimen de comunidad de bienes en el matrimonio. En consecuencia, la falta de titularidad de los activos entre las mujeres, afecta su independencia económica y la oportunidad de trabajar por cuenta propia. Según encuestas realizadas por Global Findex el 26\% de las mujeres de los países en desarrollo, en comparación con el $20 \%$ de los hombres, afirma no tener una cuenta bancaria porque tienen la posibilidad de utilizar la cuenta que posee un miembro de la familia (Demirguc-Kunt et al. 2018).

Como puede observarse, el problema al que se enfrentan las mujeres no solo abarca el no contar con una cuenta bancaria, sino que esta desventaja respecto de los hombres, se extiende también al ahorro y a la obtención de préstamos en instituciones formales. La misma fuente de datos estima que en América Latina, el 8\% de las mujeres informo haber ahorrado de manera formal el año pasado, en comparación con el 12\% de los hombres. Esta distorsión hace más profunda la dificultad de obtener información relevante para las instituciones, debido a que el comportamiento de ahorro formal es un indicador de capacidades financieras, que permite a los proveedores de servicios financieros obtener un registro de datos para conocer el perfil de riesgo del cliente e información para el otorgamiento de préstamos.

No obstante, es relevante destacar la capacidad de ahorro de las mujeres de bajos recursos que no cuentan con acceso al ahorro formal y seguro, debido a factores limitantes de tiempo, niveles de alfabetización y movilidad; que quienes, a pesar de generar escasos e irregulares ingresos, se estima que destinan entre un $10 \%$ y un $15 \%$ al ahorro (Women's World Banking 2015).

En tercer término, desde el lado de la oferta encontramos a la tecnología y servicios digitales como impulsores claves hacia el camino de la inclusión 
financiera. Su contribución permite el acceso a servicios financieros de bajo costo y estimula el uso de cuentas bancarias, por lo que la falta de disponibilidad y familiarización con el mundo de las aplicaciones móviles y las finanzas digitales abre un nuevo obstáculo a enfrentar.

Dada la relevancia de los servicios digitales en el campo de las finanzas, en el 2016 el G-20 publicó el mencionado informe impulsado por el Grupo Banco Mundial y el Banco Popular de China (GPFI 2016) en que se incluyeron ocho recomendaciones dirigidas a los países para promover la inclusión financiera utilizando las tecnologías digitales. La inclusión financiera digital, representa una ventaja a la hora de atraer y bancarizar a los a 1.500 millones de adultos excluidos del sistema financiero formal y regulado.

Si se analiza el acceso a las tecnologías desde la óptica de la brecha de género, se estima según la Asociación GSM (GSMA) que, en comparación a los hombres, hay 200 millones menos de mujeres que tienen un teléfono móvil en los países de ingreso bajo y medio. La brecha de género existente se extiende entonces al campo de internet móvil y servicios de dinero móvil, reduciendo las oportunidades comerciales y socioeconómicas de la mujer.

Otro factor causante de la exclusión del sector financiero es la falta de formación y educación financiera. Entendiéndose esta como el proceso por el cual los consumidores/inversionistas financieros mejoran su comprensión de los productos financieros, los conceptos y los riesgos, y, a través de información, instrucción y/o el asesoramiento objetivo, desarrollan las habilidades y confianza para ser más conscientes de los riesgos y oportunidades financieras, tomar decisiones informadas, saber a dónde ir para obtener ayuda y ejercer cualquier acción eficaz para mejorar su bienestar económico (OECD/INFE 2013).

Abstrayendo este limitante a la brecha de género encontramos que en promedio las mujeres tienen un menor nivel de conocimientos financieros que los hombres (OECD/INFE 2013). Las bajas capacidades financieras promueven la utilización de mecanismos informales de ahorro, la pérdida de oportunidades de inversión o estrategias de inversión erradas con escaso retorno o excesivo riesgo, provocando efectos negativos tanto en los individuos como en el bienestar de los hogares.

Las limitaciones de la capacidad de las mujeres para trabajar fuera del hogar, relacionarse con agentes masculinos, carencia de educación 
financiera o incluso poseer un teléfono móvil, son barreras que obedecen a reglas y comportamientos sociales y culturales. Los mecanismos utilizados por la banca tradicional para abordar este tipo de barreras no se ajustan a las necesidades de las mujeres, dejándolas excluidas del sistema financiero y de los beneficios que el uso efectivo que los productos financieros podría proporcionarles.

La falta de acceso impide a las mujeres tener igualdad de oportunidades en la sociedad, es por ello la creciente necesidad de desarrollar nuevos conocimientos y enfoques, en el que se contemplen y rediseñen las practicas actuales en materia de inclusión financiera para poder así reducir y finalmente cerrar las disparidades generadas en el sector a razón de la brecha de género.

Como se dijo, este trabajo se entenderá a la inclusión financiera como la creación, promoción y regulación de un ambiente financiero accesible, asequible y seguro para el conjunto de la sociedad. Tiene como fin promover el bienestar económico y la inclusión social a través de la oferta de servicios y productos financieros diseñados para satisfacer las necesidades de distintos sectores de la población (Carballo 2018)

No obstante, este concepto demuestra una gran amplitud con unos objetivos sumamente abarcativos. Posicionándose la presente revisión dentro de este marco y reconociendo la misión amplia de Inclusión Financiera, se hará foco en las microfinanzas, es decir a los "servicios financieros ofrecidos a todos aquellos que están excluidos del sistema financiero formal" (Lacalle et al. 2010). Se reconoce dentro de las microfinanzas servicios más allá de los tradicionales microcréditos, como microahorros y microseguros, entre otros (Lacalle et al. 2010; Pomeranz 2014).

En los hechos, al hablar de microfinanzas se hace referencia a agentes en situación de exclusión producto de su condición de pobreza o vulnerabilidad. Dado que no toda la literatura que aborda la presente temática mide su efecto en el empoderamiento de la mujer, el presente trabajo mencionará especialmente a dos de estos servicios: microcréditos y microahorros. Pero previo a ello, bosquejaremos un resumen de políticas para la inclusión y la definición de empoderamiento de la mujer. 


\section{II.2 Beneficios y políticas para la inclusión financiera de la mujer}

Reconocido el campo de los desafíos que enfrentan las metas de inclusión financiera de la mujer y las brechas de género existentes, es relevante mencionar las oportunidades y beneficios teóricas que el acceso a servicios financieros presenta en el camino hacia el empoderamiento de la mujer.

El mejoramiento de la situación económica de las mujeres mediante el acceso a recursos financieros le permitiría ampliar, diversificar, mejorar la calidad de su producción y aumenta la productividad, teniendo efectos directos sobre sus familias en términos de reducción de la pobreza y un mejor futuro para los hijos, ya que con los ingresos generados se pueden alcanzar mejoras sociales como el acceso a servicios básicos (salud, agua potable, saneamiento y educación), en otras palabras mejora la seguridad económica. A su vez, el acceso a opciones de ahorro y créditos formales, no solo garantizaría una mayor participación de la mujer en la economía, sino que le permitiría utilizar estas oportunidades como trampolín para salir de la pobreza y lograr una mayor igualdad (Banco Mundial 2012).

En primer lugar, se resalta que cuando aumenta la participación de las mujeres dentro de la fuerza laboral se produce un crecimiento económico más rápido. Es por ello que se incluyó dentro de los objetivos de la Agenda 2030 para el Desarrollo Sostenible, adoptada por las Naciones Unidas en 2015, la creación de más y mejores empleos para las mujeres, como así también la eliminación de la discriminación a razón de la igualdad de género tanto en el hogar como en el trabajo.

Según el Informe "Igualdad de Género y Desarrollo" (Banco Mundial 2012) se estimó que las pérdidas de ingresos debido a la exclusión de las mujeres del mundo laboral oscilan entre el $10 \%$ y el $37 \%$ del PBI en todas las regiones. Estudios del Grupo Banco Mundial, el Fondo Monetario Internacional (FMI), la Organización para la Cooperación y el Desarrollo Económicos (OCDE), y del sector privado indican que miles de millones se podrían agregar al PIB mundial con el fomento de la igualdad de la mujer.

Un estudio realizado por Mckinsey Global Institute (Woetzel et al. 2015) estima que si se cerrara la brecha de género se añadirían 28 billones de dólares al PIB mundial en 2025, equivalente a la suma de las economías de Estados Unidos y China. El escenario que contempla estos potenciales 
beneficios, realiza sus estimaciones sobre el supuesto que todas las mujeres desempeñan un papel idéntico al de los hombres en los mercados laborales.

Las políticas activas en el mercado de trabajo que buscan principalmente aumentar la participación de la mujer en el mercado laboral, son un primer paso a lograr la inclusión financiera, a través del otorgamiento de recursos económicos y mejorando la capacidad de toma de decisiones en el hogar. Según la OIT (2006), las mujeres contribuyen en mayor proporción con sus ingresos al hogar que los hombres, dando como resultado mejoras en calidad de vida de las futuras generaciones.

Asimismo, estas políticas deben ser acompañadas de la eliminación del trato discriminatorio en las leyes y normas laborales que restringen las capacidades de acción y decisión de las mujeres en el ámbito laboral, financiero y social, situación que se observa tanto en economías en desarrollo como desarrolladas. La disolución de estos prejuicios en leyes, reglamentaciones e insuficiencias en las estructuras jurídicas, lograría ampliar las oportunidades de las mujeres otorgándoles así el acceso al control de recursos del hogar.

La evolución hacia la igualdad de género contribuiría a un mejor funcionamiento del hogar y transformándose en un factor determinante en el crecimiento económico y en el empoderamiento de la mujer. Es por ello que la importancia del acceso a servicios financieros que generan una puerta hacia el crecimiento de las mujeres en el mercado laboral, principalmente como canal hacia posibilidades de trabajo independiente y potenciales emprendimientos protagonizados por mujeres, permitiría al menos teóricamente enfrentar barreras tan arraigadas en la sociedad.

Por otro lado, la mujer que tome un rol clave en el desarrollo de emprendimientos, obtendría un mayor control de sus vidas, aumentando su capacidad en la toma de decisiones, su independencia económica y el ejercicio de sus derechos. Lograr desde la inclusión financiera mayores oportunidades en este campo, generaría un impacto visible y profundo con efectos multiplicadores a nivel personal, familiar y en la sociedad. Al romper la dependencia económica dentro del hogar, la mujer adquiriría un rol importante y nuevas de herramientas que le permitirán romper con el círculo de pobreza generacional. El incremento de ingresos, beneficia como vimos anteriormente el consumo del hogar, invirtiéndose en alimentos, salud, educación y vivienda. 
La lógica del emprendedorismo y la inclusión financiera afirma que dichos emprendimientos no pueden crecer por si solos, deben ser acompañados por políticas económicas y sociales, programas de desarrollo y acceso a servicios financieros. El principal reto es lograr la sinergia entre los distintos actores del sector público, privado y de sociedad civil, quienes a través de los distintos recursos y capacidades puedan lograr mayores impactos garantizando el pleno derecho de las mujeres.

En lo relativo a las dificultades ya mencionadas de las mujeres para acceder al ahorro formal y seguro y a los limitantes que enfrentan en materia de tecnología, encontramos como desde la óptica de la inclusión financiera, existen mecanismos de ahorro digital. Esta opción segura y de bajo costo, les permitiría a las mujeres ahorrar pequeñas cantidades de dinero utilizando canales alternativos como corresponsales bancarios y dinero móvil. Sin embargo, aún no se ha desarrollado un modelo integral de servicios financieros digitales que efectivamente sirva y se implemente a mujeres de bajos ingresos en la escala. Esto viene en parte, a consecuencia de la falta de educación financiera y asesoramiento en la materia.

Las mujeres también serían empoderadas a través de la capacitación técnica y financiera, para ello es necesario contar con programas de coordinación entre el sector bancario y público. Incentivar y promover la educación financiera permitiría generar nuevas oportunidades y opciones para elevar el nivel de vida de las mujeres, siendo el manejo de sus finanzas un factor clave para optimizar la gestión de su dinero.

Tras haber definido los causales teóricos comúnmente señalados en torno a las bondades de promover la inclusión financiera de las mujeres, definiremos el concepto de Empoderamiento de la mujer.

\section{II. ¿Qué es el empoderamiento de la mujer?}

El primer paso para evaluar el impacto de una variable sobre otra es conceptualizar a ambas. Ya hemos definido la inclusión financiera y sus puentes teóricos con la equidad de género. Por su parte, el término "empoderamiento" es ampliamente utilizado, pero debe explicitarse a qué se hace referencia concretamente con ello y cuáles son las notas que lo definen, lo cual nos acercará a una posible medición de este fenómeno. 
Se destacan varias definiciones, pero todas hacen un punto común en el concepto "agencia". La importancia de ello yace en el rechazo a abordajes descendentes o "top-down". Es que, en el empoderamiento, tan importante como la agencia es el proceso (Narayan-Parker 2005).

Kabeer define al empoderamiento como "la expansión en las posibilidades de las personas para realizar elecciones estratégicas en sus vidas en un contexto donde esta capacidad se les había sido previamente denegada" (Kabeer 2009). La definición destaca la capacidad del individuo de poder elegir (agencia), así como en el proceso de cambio hasta lograr esta capacidad.

Siguiendo a Glennerster y coautores (2018), una definición en este sentido permite operacionalizar al empoderamiento en tres dimensiones:

- Recursos: acceso a recursos material, humanos y sociales. Ello resalta las capacidades de las personas para poder ejercer una elección.

- Agencia: rol activo en el proceso de elección de decisiones para su vida.

- Logros: mejoras destacadas en la vida y bienestar a raíz del aumento en la agencia.

Por otra parte, el empoderamiento se encuentra enmarcado en un contexto (sociocultural, político, económico), por lo que es moldeado por la interacción entre normas e instituciones (Glennerster et al. 2018). El concepto resultante solo tiene significado en ese contexto específico (Narayan-Parker 2005). No obstante, deben seguirse ciertos estándares universales, como los establecidos en convenciones internacionales.

El concepto del empoderamiento puede entonces ser aplicado a cualquier grupo excluido o en desventaja, pero el empoderamiento de la mujer tiene algunas notas distintivas. Por un lado, es un grupo trasversal a todos, con el alcance que ello implica. Por otra parte, cobra especial relevancia las relaciones intrafamiliares (Narayan-Parker 2005).

\section{II.4 Observaciones sobre la medición empoderamiento}

Como se destacó, el empoderamiento es un proceso, no una condición o estado. Por ende, los recursos empíricos por captarlos suelen ser complejos.

El empoderamiento no puede medirse directamente sino a través de indicadores o proxis. Éstos son multidimensionales, variando según el 
enfoque del estudio, pero siguiendo el foco que anteriormente se mencionó: agencia, proceso, contexto. Esto implica que las mujeres pueden empoderarse en un área y en otras no.

Por ello debe tenerse en cuenta que una intervención puede promover el empoderamiento sobre una dimensión y que no necesariamente también lo hará en otra. No obstante, las variables de distintas dimensiones suelen estar relacionadas: por ejemplo, es difícil, lograr un completo empoderamiento económico sin el empoderamiento en la esfera familiar.

Sin embargo, debe tenerse cuidado en los indicadores elegidos. Varios estudios utilizan proxis como la salud y la educación, pero estas variables pueden verse afectadas por otras que no correspondan a elecciones por presencia (ausencia) de empoderamiento. Como respuesta se han aumentado los esfuerzos por capturar procesos a través de indicadores que capten el poder de decisión y elección de las mujeres, que sean muy concretos (Narayan-Parker 2005).

En la actualidad la forma con más poder explicativo para esta medición son los experimentos controlados aleatorios. En este tipo de pruebas, a los individuos se los asigna de forma aleatoria a un programa de microfinanzas determinado (grupo tratamiento) o no (grupo de control). La aleatoriedad hace que, en promedio, los grupos sean iguales (Glennerster et al. 2018).

El problema con los estudios no aleatorios, que comparan individuos que han tomado un servicio financiero con individuos que no, es que la muestra puede estar sesgada: los individuos que han, por ejemplo, tomado un crédito, tengan menos aversión al riesgo, mayores capacidades para usarlos, etc. que puede que los hagan más prósperos, incluso antes de comenzar el programa. La aleatoriedad elimina este problema. Si bien las pruebas aleatorias sirven para establecer causalidad, solo aplican al contexto particular de la evaluación (Bauchet et al. 2011).

No obstante, este tipo de estudios se han comenzado a aplicar desde la década pasada, por lo que la literatura previa ha utilizado otros métodos, que por ejemplo no son aleatorios. A continuación, realizaremos un análisis de la literatura empírica en torno a la inclusión financiera y su impacto en el empoderamiento de la mujer. 


\section{Evidencia de dirigir productos microfinancieros a las mujeres}

Tener como objetivo a las mujeres como participantes de programas de inclusión financiera en la pobreza, o programas microfinancieros, tienen un doble beneficio según la literatura. Por un lado, como se dijo, la evidencia demuestra que son mejores pagadoras. Por otro, tendría mayores efectos positivos sociales que si se destinase a los hombres producto del rol de administradoras del hogar (Narayan-Parker 2005).

Muchos programas microfinancieros se han implementado, en especial en países en desarrollo, cuyas destinatarias son mujeres, en especial aquellos cuyo servicio son los microcréditos. Además de la evidencia en torno a su mejor desempeño a la hoja de los repagos, la misma también señala que las mujeres suelen estar más restringidas en cuanto al acceso al crédito, al trabajo asalariado y además tienen una distribución inequitativa del "trabajo no remunerado" (Pitt y Khander 1998).

Dado un incremento en el ingreso de las mujeres, el gasto destinado a bienes de consumo en el hogar y al bienestar de sus hijos aumenta, mientras que en caso de darse en los hombres, no se observa el mismo impacto (Duflo y Udry 2004). Es decir, hay una diferencia entre géneros ante un mismo shock en sus ingresos en cuanto a los efectos del mismo.

Como se señaló anteriormente, parte esencial del proceso de empoderamiento es el poder ganar control para poder elegir. Las mujeres, en especial en contextos vulnerables y culturas patriarcales, suelen tener menos poder de decisión sobre elecciones que versen sobre su vida y la de su hogar. Esto se relaciona con el poder de fungibilidad que tiene el dinero dentro del hogar, el cual varía entre contextos. De la evidencia surgida al respecto de este tema surge la necesidad de crear productos que se ajusten a esta necesidad y contextos específicos, previendo estas dinámicas intrahogareñas a la hora de evaluar su impacto en el empoderamiento femenino.

Por ejemplo, Mani (2011) señala que el acceso a la información en estas parejas no afecta la eficiencia a la hora de hacer inversiones: al aumentar la cantidad de ingresos generados (y por ende su proporción en el total del hogar) por la mujer, sus esposos están más dispuestos a realizar inversiones que reduzcan sus propios ingresos, pero que también "castiguen" a sus esposas, de manera tal que puedan seguir manteniendo el control del flujo. 
La autora señala que esta podría ser una de las razones detrás de que los emprendimientos liderados por mujeres suelan tener menores retornos (Mani 2011).

En culturas donde los hombres tengan el poder de decisión financiero dentro del hogar y controlen el monto provisto a sus esposas, los medios informales de ahorros informales proveen a las mujeres una opción para resguardar esto en forma de ahorro; mientras que en aquellas donde las normas dicten que el hombre debe dar a su esposa el dinero que genere ya que ella es la administradora financiera del hogar, ellos tendrán más incentivos a esconder parte de sus ingresos (Ahraf, 2009).

III.1 Observaciones de la literatura pre-experimentos controlados aleatorios

\section{III.1.1 En la mujer}

Schuler et al. (1996) mostraron efectos positivos en el impacto causado por los microcréditos tomados por mujeres en su bienestar y estatus, incluso en aquellos casos donde las mujeres no controlan los desembolsos. Aumenta su confianza en sí mismas y estatus dentro de su familia como proveedoras de ingresos que ayuden al hogar (Goetz y Sengupta 1996).

Las mujeres que se han enrolado en programas de crédito mostraron tener un rol mayor dentro de la toma de decisiones del hogar así como vis-a-vis con sus esposos, un mayor acceso a recursos económicos y financieros, más grandes redes sociales y una mayor comunicación intramarietal sobre planificación familiar y crianza de sus hijos. Contrariamente, si son los hombres los destinatarios de los montos de estos programas, su efecto en el empoderamiento de la mujer suele ser negativo, mostrando efectos negativos en todos estos indicadores (Pitt et al. 2006).

\section{III.1.2 En su familia}

En los hogares donde mujeres reciben créditos, se demuestran mayores ingresos y nivel de consumo que aquellos que no lo han recibido. No obstante, estos indicadores mejoran más entre los hombres que en las mujeres (Khan 1999). 
Al mejorar el empoderamiento de la mujer mejora la tasa de supervivencia de los niños, la tasa de escolaridad y el gasto destinado a su salud y educación (Joshi 2004; Lancaster et al. 2003; Durrant y Sathar 2000).

Parte de la literatura ha buscado comprobar si existe algún tipo de diferencia por sexo en los efectos que genera el aporte de recursos a los hogares. En este sentido, existe evidencia de que la magnitud del impacto del crédito en el bienestar de los niños que lo conforman depende del género del prestatario (Pitt y Khandker, 1998; Khandker et al., 1995; McKernan, 2002; Pitt et al., 2003 y 2006).

III.1.3 Control sobre el crédito: evidencia negativa sobre el empoderamiento

Parte de la literatura ha dedicado esfuerzos en problematizar la capacidad transformadora de los microcréditos.

Una parte de la inclusión financiera es el acceso al crédito, problema al cual múltiples instituciones se han dedicado a resolver ideando productos que se ajusten a la realidad de las mujeres, solucionando problemas como la falta de garantías con sistemas como créditos grupales.

No obstante, aparece un problema de segundo orden que es el "control" de los recursos obtenido que aborda el grado de fungibilidad del crédito entre parejas (heterosexuales) (Pitt y Khander 1998).

Si bien el acceso al microcrédito aumenta el flujo de caja en los hogares, en ausencia de cambios en las relaciones entre géneros dentro del hogar, la distribución y uso del dinero permanece mayormente en el hombre fortaleciendo su posición (Montgomery et al. 1996; Kabeer 2009).

Este fenómeno también se explica porque invertir montos relativamente grandes requiere conocimientos, información, habilidades contables y de gestión que en muchos contextos suele tener el hombre más que la mujer (Khan 1999).

Mientras tanto, son las mujeres quienes mantienen la obligación contractual del repago, llegando a recurrir a ingresos destinados al ahorro o al consumo de bienes básicos (Goetz y Gupta 1996).

La mejora en el status y reconocimiento dentro del seno familiar es limitada. Incluso este ingreso es percibido como fuente de tensiones ante la 
posibilidad de incumplimiento y consecuente venta de activos (Montgomery et al. 1996; Mayoux 2002), manifestándose un aumento en la violencia de género (Goetz y Gupta 1996; Mayoux 2002).

Debido a que es usual que sean otros quienes usen el dinero obtenido a través de un crédito, las mujeres en situación de pobreza prefieren el trabajo asalariado sobre emprender con un crédito, nos sólo por su estabilidad y posibilidad de socialización por fuera del seno familiar (y evitar posibles conflictos o situaciones de violencia), sino porque se sienten menos alienadas a este tipo de ingreso (Khan 1999). Ello no significa que no requieran además servicios financieros

Mayoux (2002) señala que aún en programas de microfinanzas exitosos, la contribución de estas al empoderamiento de la mujer es limitado: las mantiene en trabajos de bajos ingresos, una mayor carga de trabajo ya que suman el remunerado a las tareas no remuneradas, la prioridad en sus gastos serán siempre sus hijos.

Asimismo, en el caso de los créditos grupales existe un doble problema: por un lado consume el tiempo (tanto de ocio como de trabajo) de las mujeres y las presiones para el repago pueden llevar a una exclusión mayor, en este caso, entre las propias mujeres (Mayoux 2002).

Por otro lado, a las mujeres muchas veces se las confina a créditos de bajo monto, ya que suelen haber prejuicios tanto en la elaboración del programa como en los agentes, aun en casos donde las mujeres tienen y interés y la capacidad de afrontar créditos mayores (Mayoux 1998).

\section{III.2 Estudios randomizados y efectos en el empoderamiento de la mujer}

Como se dijo, en la actualidad la forma con más poder explicativo para la medición de impacto son los experimentos controlados aleatorios. En este tipo de pruebas, a los individuos se los asigna de forma aleatoria a un programa de microfinanzas determinado (grupo tratamiento) o no (grupo de control). La aleatoriedad hace que, en promedio, los grupos sean iguales (Glennerster et al. 2018). 


\section{III.2.1 Microcréditos}

Angelucci, Karlan y Zinman (2013) realizaron un estudio aleatorio en clusters en asociación con Compartamos, IMF que provee microcréditos productivos a mujeres bajo modalidad grupal durante tres años. Sus conclusiones fueron que los microcréditos demostraron crecimientos modestos en del tamaño de los emprendimientos, confianza y poder decisión en sus hogares, y en la misma magnitud en disminuciones de depresión y necesidad de ayuda/asistencia. No obstante, hay poca evidencia de efectos dañinos. No hay efectos transformativos en medidas como ganancias, ingresos o consumos mayores.

Banerjee, Duflo, Glennerster y Kinnan (2015) en asociación con Spandana (una de las IMF más importantes de India realizaron un estudio controlado entre 2005 y 2010 sobre 52 comunidades de Hyderabad elegidas aleatoriamente, donde al grupo de tratamiento se le otorgó créditos grupales destinados a mujeres. Esta investigación no demostró un aumento en el empoderamiento de las mujeres, entendido como poder de negociación, para el cual elaboraron un índice con 16 proxis que incluyen indicadores que versan sobre el control de los gastos e inversiones tanto en bienes para el hogar como en salud y educación para sus hijos.

El estudio mostró que hubo un aumento en la probabilidad de emprender un negocio en $1,7 \%$ en comparación con las áreas de control. Además, si bien no se mostró un aumento en el consumo del hogar, virando hacia el consumo de bienes durables, recortes en "bienes de tentación". Crépon, Devoto, Duflo, y Pariente (2015) realizaron un estudio de 2006 a 2010 a en Marruecos en donde la IMF Al Amana planificaba expandir sus operaciones. La evaluación versó sobre 162 villas, divididas en 81 pares de características similares, ofreciendo créditos grupales en una de cada par de forma aleatoria.

Estos créditos no fueron otorgados exclusivamente a mujeres, sino que en su mayoría fueron hombres. No obstante, se procedió a la construcción de un índice sobre una serie de variables cualitativas que describen al empoderamiento femenino, hallando que no hay efectos positivos de las microfinanzas sobre éste. Los autores explican que este resultado es consistente con la evidencia de que una proporción muy pequeña de mujeres 
rurales piden créditos y que los que son concedidos a hombres no cambia la distribución de poder intrafamiliar.

\section{III.2.2 MicroAhorro}

Dupas y Robinson (2013a y b) llevaron a cabo un estudio en Kenia en colaboración con el banco local de Bumala donde proveyeron de forma aleatoria a pequeños comerciantes por primera vez de cuentas de ahorro que no otorgaban ningún tipo de interés y tenían altos costos a los retiros (siendo aun así una opción mejor a las alternativas que tenían). Además, se los instó a detallar diariamente inversiones, gastos y shocks de salud.

El 40\% de quienes decidieron tomar estas cuentas con tasas de interés negativas fueron mujeres, quienes las usaron de forma más intensa que los hombres. Estos ahorros a su vez llevaron a otros efectos positivos, como que entre los 4 y 6 meses posteriores a la apertura de la cuenta, las mujeres tenían un $45 \%$ más de inversión diaria que el grupo de control femenino. También aumentaron sus gastos en bienes de consumo para ellas y sus hijos (por ejemplo, el gasto en comida aumentó entre un 10 y un $20 \%$.

Mostraron menos propensión a desinvertir en sus negocios debido a shocks negativos de salud (muy comunes en este contexto) y afrontar de mejor manera tratamientos médicos. También se demostró que las cuentas fueron beneficiosas para reducir el riesgo de apropiación de sus ahorros por parte de familiares, amigos, conocidos (aunque los autores recomiendan más investigación al respecto de esto debido al tamaño de su muestra).

No se mostraron efectos en los hombres (se cree porque ellos pueden ahorrar de forma más segura en sus hogares y disponen de otras alternativas). De esta forma, el potencial de los servicios de ahorro en el bienestar de las mujeres para ser mucho más contundentemente positivo que los de crédito.

III.2.3 Otros estudios sobre comportamientos financieros de las mujeres: retorno del capital

Otras series de estudios se han llevado a cabo donde si bien a la población objetivo no se le ha ofrecido ningún producto microfinanciero, sus objetivos buscan ver como las mujeres invierten, pudiendo extraer además 
conclusiones sobre su empoderamiento e idear productos que se ajusten a su realidad.

De Mel, McKenzie y Woodruff (2008) estudiaron la tasa de retorno (real) del capital de las microempresas (unipersonales) en Sri Lanka, donde se realizaron shocks de capital en forma aleatoria. Este resultó de un promedio de $60 \%$ anual (mayor que la tasa de interés del mercado), aunque con gran dispersión. Sin embargo, el género de los dueños influía en esta conclusión: la mitad de las mujeres observadas daban señales de generar tasas negativas.

En una repetición de este estudio (De Mel et al. 2009) observaron que los hombres invertían una gran proporción de estos fondos, generando retornos de entre 6,5 y $14 \%$. Contrariamente, las mujeres no invertían los fondos en sus negocios si el monto era pequeño, y de ser grade, en promedio no obtenían retornos. Los autores especulan que esto se deba al nivel de cooperación de sus esposos, apareciendo nuevamente el poder relativo de las mujeres y el nivel de control que tengan sobre sus ingresos como posible variable explicativa.

Fafchamps, McKenzie, Quinn y Woodruff (2011) llevaron a cabo un estudio similar en Ghana. Éste demostró que los hombres demostraron retornos de capital tanto en los fondos recibidos en especie como en efectivo. Por otra parte, las subvenciones en efectivo a mujeres no producían retorno del capital, mientras que, al hacerlo en especie, sí lo hacía. En el primer caso, las mujeres utilizaban una proporción de la ayuda en gastos relativos a sus hogares, que para los autores está más relacionado con una falta de "autocontrol" que con la presencia de presión en el hogar "apropiarse" de sus ingresos. Sólo las mujeres que tenían emprendimientos más grandes mostraron un mayor retorno. La recomendación surgida es que tanto las subvenciones como los créditos productivos para mujeres deben darse en especie, financiado la compra directamente con el proveedor.

\section{Conclusiones y reflexiones finales}

La literatura sobre la inclusión financiera, en especial sobre las microfinanzas, abordando como objetivo primario o secundario al empoderamiento de la mujer es frondosa. No obstante, los trabajos en cuyo método se hayan llevado a cabo experimentos aleatorizados controlados son 
de poca data. Sus conclusiones tienen un mayor poder de explicación (con las limitaciones del contexto aplicado que desde ya aplican a cualquier investigación de este tipo) y ponen en jaque a aquellas a las que se han arribado en otras investigaciones no aleatorizadas con antelación. El problema con estas últimas yace en el sesgo que plantea la selección de la muestra, donde el grupo de observación y el de control no son en promedio iguales, teniendo características de base probablemente distintas.

A pesar de que los resultados no son tan prometedores sobre el beneficio de la inclusión financiera o bien de las microfinanzas como se creía que los tenían, siguen mostrándose efectos positivos en algunos contextos, en particular en el empoderamiento de la mujer. Por su parte, el hecho de que no exista evidencia robusta y concluyente sobre un impacto positivo entre las microfinanzas y el empoderamiento de las mujeres no implica que exista sobre su impacto negativo. Cabe destacar que el estado del arte en la actualidad nos reafirma la necesidad de desarrollo de más estudios en otras culturas y sobre otros servicios para poder llegar a un nivel comparativo mucho mayor.

Un factor común a todo tipo de estudios sobre el empoderamiento, en este caso relativos a la inclusión financiera, a señalar es la importancia de las dinámicas familiares. La falta de empoderamiento de las mujeres pobres es mayor que la de sus esposos, hermanos o padres. Es que las limitaciones no sólo provienen de la falta de recursos materiales, sino por la falta de control sobre sus propias vidas, producto de una relación desigual con sus parejas (principalmente).

Esta distinción se encuentra enmarcada por el contexto social, cultural e institucional que dan más o menos poder a las mujeres. Ello cobra sentido si pensamos que lo que diferencia a los hombres de las mujeres como personas es la capacidad gestacional de estas últimas. El problema es el nivel en que cada sociedad tolera y/o avala la limitación o reclusión de las mujeres que la componen a esta distinción meramente física, imposibilitando su desarrollo en otras dimensiones. Entonces, mientras no haya una equidad en la distribución de las tareas reproductivas, lo cual requiere un abordaje multidimensional que atienda a esta diferencia, cualquier programa univariado como aquellos centrados en las finanzas tendrá un impacto limitado, algunos más que otros. 
Cualquier tipo de programa o política que busque empoderar económicamente a las mujeres no sólo debe prestar atención a que ellas puedan generar ingresos propios, sino que también puedan ser iguales frente a sus parejas. No habrá equidad ni prosperidad mientras las relaciones entre hombres y mujeres sean desiguales.

\section{Referencias}

Angelucci, M., D. Karlan y J. Zinman. 2013. Win some lose some? Evidence from a randomized microcredit program placement experiment by Compartamos Banco (No. w19119). National Bureau of Economic Research.

Ashraf, N. 2009. "Spousal control and intra-household decision making: An experimental study in the Philippines". American Economic Review 99 (4): 1245- 77.

Banco Mundial. 2012. Informe sobre el desarrollo mundial 2012: igualdad de género y desarrollo. Washington, DC: Banco Mundial.

- 2016. Mujer, Empresa y el Derecho 2016: Alcanzando la Igualdad. Washington, DC: Banco Mundial. doi: 10.1596.

- 2018. "Financial inclusion". Disponible en: https://www.worldbank.org/en/topic/financialinclusion/overview

Banerjee, A., E. Duflo, R. Glennerster y C. Kinnan. 2015. "The miracle of microfinance? Evidence from a randomized evaluation". American Economic Journal: Applied Economics 7 (1): 22-53.

Bauchet, J., C. Marshall,L. Starita, J. Thomas y A. Yalouris. 2011. "Latest findings from randomized evaluations of microfinance". FORUM 2. Disponible ${ }^{-}$ en: https://www.povertyactionlab.org/sites/default/files/publications/FORU M2.pdf

Bin-Humam, Y. 2017. "5 Challenges for Women's Financial Inclusion”. Disponible en:

https://www.cgap.org/blog/5-challenges-womens-financial-inclusion Carballo, I. E. 2017. "Financial inclusion in Latin America". En Global Encyclopedia of Public Administration, Public Policy, and Governance, 
editado por A. Farazmand. Cham: Springer International Publishing. doi: 10.1007/978-3-319-31816-5_3333-1

Crépon, B., F. Devoto, E. Duflo y W. Parienté. 2015. "Estimating the impact of microcredit on those who take it up: Evidence from a randomized experiment in Morocco". American Economic Journal: Applied Economics 7 (1): 123-50.

De Mel, S., D. McKenzie y C. Woodruff. 2008. "Returns to capital in microenterprises: evidence from a field experiment". The quarterly journal of Economics 123 (4): 1329-1372.

- 2009. "Are women more credit constrained? Experimental evidence on gender and microenterprise returns". American Economic Journal: Applied Economics 1 (3): 1-32.

Demirguc-Kunt, A., L. Klapper, D. Singer, S. Ansar y J. Hess. 2018. The Global Findex Database 2017: Measuring financial inclusion and the fintech revolution. The World Bank.

Duflo, E. y C. Udry. 2004. Intrahousehold resource allocation in Cote d'Ivoire: Social norms, separate accounts and consumption choices (No. w10498). National Bureau of Economic Research.

Dupas, P. y J. Robinson. 2013a. Savings constraints and microenterprise development: Evidence from a field experiment in Kenya. American Economic Journal: Applied Economics, 5(1), 163-92.

. 2013b. "Why don't the poor save more? Evidence from health savings experiments". American Economic Review 103 (4): 1138- 1171.

Durrant, V. L. y Z. Sathar. 2000. "Greater investments in children through women's empowerment: A key to demographic change in Pakistan". Working Paper No. 137. Policy Research Division, Population Council, USA.

EIU. 2019. Global Microscope 2019: The Enabling Environment for Financial Inclusion.

Fafchamps, M., D. McKenzie, S. Quinn y C. Woodruff. 2011. When is capital enough to get female enterprises growing? evidence from a randomized experiment in Ghana. The World Bank.

Glennerster, R., C. Walsh y L. Diaz-Martin. 2018. A Practical Guide to Measuring Women's and Girl's Empowerment in Impact Evaluations. JPAL. Disponible en: 
https://www.povertyactionlab.org/sites/default/files/resources/practicalguide-to-measuring-womens-and-girls-empowerment-in-impactevaluations.pdf

GPFI. 2016. Principios de Alto Nivel sobre la Inclusión Financiera Digital - Grupo Banco Mundial. Disponible en: http://www.gpfi.org/news/newg20-high-level-principles-digital-financial-inclusion

Goetz, A. M. y R. S. Gupta. 1996. "Who takes the credit? Gender, power, and control over loan use in rural credit programs in Bangladesh". World development 24 (1): 45-63.

Joshi, S. 2004. "Female household-headship in rural Bangladesh: incidence, determinants and impact on children's schooling". Yale University Economic Growth Center Discussion Paper 894.

Kabeer, N. 2009. "Conflicts over credit: re-evaluating the empowerment potential of loans to women in rural Bangladesh". En Microfinance, págs. 128-162. Routledge.

Khan, M. R. 1999. "Microfinance, wage employment and housework: a gender analysis". Development in practice 9 (4): 424-436.

Khandker, S. R., M. B. Khalily y Z. H. Khan. 1995. Grameen Bank: performance and sustainability (Vol. 306). World Bank Publications.

Lacalle, M., S. Rico, J. Márquez, B., I. Jiménez, M. D. C. de la Orden y A. González. 2010. "Glosario básico sobre microfinanzas" (reedición ampliada). Fundación Nantik Lum de Microfinanzas. Cuadernos Monográficos 12: 146.

Lancaster, G., P. Maitra y R. Ray. 2003. "Endogenous Power, Household Expenditure Patterns and Gender Bias: Evidence from India". Monash University Department of Economics Working Paper (20-03).

Mani, A. 2011. "Mine, your or ours? The efficiency of household investment decisions: an experimental approach". University of Warwick. Working Paper. Unpublished

Mayoux, L. 1998. "Participatory Learning for Women's Empowerment in Micro-Finance Programmes: Negotiating Complexity, Conflict and Change". IDS bulletin 29 (4): 39-50.

- 2002. "Microfinance and women's empowerment: Rethinking 'best practice"'. Development Bulletin 57 (76-80). 
McKernan, S. M. 2002. "The impact of microcredit programs on selfemployment profits: Do noncredit program aspects matter?" Review of economics and statistics 84 (1): 93-115.

Montgomery, R., D. Bhattacharya y D. Hulme. 1996. Credit for the poor in Bangladesh. The BRAC rural development programme and the government Thana resource development and employment programme.

Narayan-Parker D. 2005. Measuring Empowerment: Cross-Disciplinary perspectives. The International Bank for Reconstruction and Development. The World Bank. Washington, DC.

OECD/INFE. 2013. Women and Financial Education Evidence, Policy Responses and Guidance. Disponible en:

https://www.oecd.org/daf/fin/financial-education/women-and-financialeducation-2013.htm

ONU Mujeres. 2018. Turning promises into action: Gender equality in the 2030 Agenda for Sustainable Development. New York: UN Women. Consultado el 8/12/18: www.unwomen.org/sdg-report

Pitt, M. M. y S. R. Khandker. 1998. "The impact of group-based credit programs on poor households in Bangladesh: Does the gender of participants matter?" Journal of political economy 106 (5): 958-996.

Pitt, M. M., S. R. Khandker y J. Cartwright. 2006. "Empowering women with micro finance: Evidence from Bangladesh". Economic Development and Cultural Change 54 (4): 791-831.

Pitt, M. M., S. R. Khandker, O. H. Chowdhury y D. L. Millimet. 2003. "Credit programs for the poor and the health status of children in rural Bangladesh". International Economic Review 44 (1): 87-118.

Pomeranz, D. D. 2014. "The promise of microfinance and women's empowerment: what does the evidence say?" EY Thought Leadership Series (a).

Schuler, S. R., S. M. Hashemi, A. P. Riley y S. Akhter. 1996. "Credit programs, patriarchy and men's violence against women in rural Bangladesh". Social science \& medicine 43 (12): 1729-1742.

Woetzel, J., A. Madgavkar, K. Ellingrud, E. Labaye, S. Devillard, E. Kutcher y M. Krishnan. 2015. The power of parity: How advancing women's equality can add $\$ 12$ trillion to global growth. McKinsey Global Institute. 
Women's World Banking. 2015. "Digital savings: The key to women's financial inclusion?" Disponible en:

https://www.womensworldbanking.org/wp-

content/uploads/2015/08/Digital-Savings-The-Key-to-

Women $\%$ E2\%80\%99s-Financial-

Inclusion_WomensWorldBanking.pdf 